\title{
Transient Diabetes Insipidus Following Organophosphorus Poisoning
}

\author{
Niraj Kumar Keyal*, Amid Bhujel \\ Department of Critical Care Medicine, B \& C Medical College and Teaching Hospital and Research Center, Birtamode, \\ Nepal
}

\begin{abstract}
Introduction: Organophosphorus poisoning is the most common poison used for suicidal attempt in Nepal. Diabetes insipidus is unusual and rare in this poisoning. This is the second case report of Diabetes insipidus developing in organophosphorus poisoning. Management of diabetes insipidus includes desmopressin and adequate fluid management.

Case presentation: A 34-year-old female patient accompanied by her father presented at the Emergency department with an alleged history of ingestion of unknown amount of chlorpyrifos, cypermethrin and quinalphos. On admission, she had a Glasgow Coma Scale (GCS) of 7/15. Her blood pressure was $110 / 60 \mathrm{~mm} \mathrm{Hg}$, pulse 54/min, respiratory rate $45 / \mathrm{min}$ and saturation $35 \%$ on room air, pinpoint pupil reactive to light and bilateral crepitations. She was immediately resuscitated with two litres of normal saline and intubated with a $7 \mathrm{~mm}$ endotracheal tube. Atropinisation was done, and pralidoxime was started. She developed a urine output of 250-350 ml per hour with rising sodium and serum osmolality. The urine examination showed low sodium and urine specific gravity. A diagnosis of diabetes insipidus was made. There was no immediate improvement in her GCS. She was managed with $5 \%$ dextrose and subcutaneous desmopressin and was transferred out of the intensive care unit on the sixth day and was discharged from hospital on the fifteenth day.
\end{abstract}

Conclusion: Diabetes insipidus is a rare transient complication in organophosphorus poisoning that requires careful observation and early management with desmopressin and adequate fluid balance to improve patient outcome.

Keywords: diabetes insipidus, desmopressin, organophosphorus, urine

Received: 10 July 2019 / Accepted: 23 September 2019

\section{INTRODUCTION}

Organophosphorus (OP) poisoning with diethyl phosphoryl chloride compound is most common over the counter poison available in Nepal. [1-3] It causes acetylcholinesterase inhibition leading to acute cholinergic crisis and manifests as nicotinic, muscarinic and central nervous system effects [4].

Antidiuretic hormone $(\mathrm{ADH})$ is synthesized at the supraoptic and paraventricular nuclei and released in response to plasma osmolality and circulating blood volume and act at collecting tubules to absorb water and to maintain osmolality and blood volume. Diabetes insipidus (DI) can be central or nephrogenic and occurs due to the absence, decrease, or unresponsiveness of $\mathrm{ADH}$. Diabetes insipidus (DI) is a rare and unusual complication of OP poisoning. Previously, a case report of DI following malathion poisoning has been reported [5], but this is the first case report of DI as a result of diethyl compound ingestion.

\section{CASe Presentation}

A 38 years old female weighing sixty kilograms, without any significant past medical or surgical history, accompanied by her father, presented at the emergency department of the General Superspeciality Hospital, Birtamode, Nepal after being referred by a primary physician from a local hospital. She had ingested an unknown amount of chlorpyrifos, cypermethrin and quinalphos, six hours previously, allegedly to commit suicide.

At the time of admission to the emergency department, her GCS was $7 / 15$, and her pupils were pinpoint reactive to light. Her pulse rate was 54 beats per min, blood pressure $110 / 60 \mathrm{mmHg}$, respiratory rate 45 breaths/min and oxygen saturation $35 \%$ on 15 litres of oxygen. Chest bilateral crepitations and wheezes were present on chest auscultation. Cardiovascular and abdominal examinations were normal. Arterial blood gas analysis showed $\mathrm{pH} 7.35$, partial pressure of oxygen $\left(\mathrm{PaO}_{2}\right) 46 \mathrm{~mm} \mathrm{Hg}$; 
partial pressure of carbon dioxide $\left(\mathrm{PaCO}_{2}\right) 31 \mathrm{~mm} \mathrm{Hg}$; lactate six mmol/L and bicarbonate $18 \mathrm{mEq} / \mathrm{L}$. A diagnosis was made of Type 1 respiratory failure with metabolic and lactic acidosis.

An emergency physician and paramedics immediately resuscitated her by inserting a 16 gauze cannula, central venous catheter and giving two litres of normal saline. Intubation was undertaken with $7 \mathrm{~mm}$ endotracheal tube.

The Peradeniya Organophosphorus Poisoning (POP) scale was 8 . Gastric lavage was achieved with sixty grams activated charcoal and two litres of normal saline, sevenhour after the ingestion of the organophosphorus compound.

An atropine challenge test was carried. One $\mathrm{ml}$ of atropine being positive which was evidenced by an increase in heart rate by more than 25 beats per minute, from 58 to 90 beats per minute, and atropinisation was attained at $50 \mathrm{ml}$ of atropine and atropine infusion was started at $5 \mathrm{ml} /$ hour. Pralidoxime chloride (Troikaa Pharmaceuticals Ltd., Ahmedabad, India) was started at one gram every six hours, according to the hospital protocol.

Her investigation profiles were total leucocyte count (TLC) - $13000 / \mathrm{mm}^{3}$; platelets- $140000 / \mathrm{mm}^{3}$; haemoglobin $(\mathrm{Hb})-9 \mathrm{gm} / \mathrm{dl}$; urea-58 mg/dl; creatinine- $1.3 \mathrm{mg} /$ $\mathrm{dl}$; sodium and potassium were $137 \mathrm{mmol} / \mathrm{L}$ and 3.9 $\mathrm{mmol} / \mathrm{L}$, respectively. Liver function test was normal except for alanine aminotransferase, which was $96 \mathrm{U} / \mathrm{L}$. A chest $\mathrm{x}$-Ray showed bilateral infiltrates. Serum acetylcholinesterase and RBC cholinesterase measurements were not obtained as these tests were not available in the hospital at the time.

The patient was managed with full ventilatory support. Atropine (Hindustan Medicines Pvt. Ltd., Barauni, India) infusion was started at $5 \mathrm{ml} / \mathrm{hr}$ intravenously, pralidoxime (Troikaa Pharmaceuticals Ltd., Ahmedabad, India) one gram intravenously every six hours, piperacillin-tazobactam (Aristo Pharmaceuticals Pvt. Ltd., Mandideeep, India) $4.5 \mathrm{~g}$ intravenously every eight hours. Routine monitoring of vital signs, input and output charting to attain a positive intake to output balance and ulcer protection with intravenous ranitidine (Cadilla Pharmaceutical, Ahmedabad, India) $50 \mathrm{mg}$ every eight hours were carried out.

She developed a urine output of 250-300 ml/hour four hours after admission to the ICU, and there was no improvement in her GCS. The urine output remained constant for the next six hours and by twenty-four hours she had passed a total of six litres.
Based on the urine specific gravity of 1.001, urine osmolality $200 \mathrm{mOsm} / \mathrm{kg}$, serum sodium $158 \mathrm{mEq} / \mathrm{L}$ and plasma osmolality $322 \mathrm{mOsm} / \mathrm{kg}$, a diagnosis of DI was made. Serum ADH was not measured.

Starting from the second hospital day, fluid management was accomplished by $5 \%$ dextrose intravenously, at a rate of $100 \mathrm{ml} /$ hour and injection. Vasopressin, six units subcutaneously was given as an initializing dose. Desmopressin was not unavailable at the hospital pharmacy during that time.

She responded briefly for two hours after which a high urine output restarted. Strict fluid input and output charting was done to avoid negative balance, and serum sodium and potassium were measured every six hours. On the following day, urine output was 7 litres/day.

Desmopressin (Sun Pharmaceutical Industries Ltd., Mumbai, India), which had been imported from India, and was started at 100 micrograms, and two puffs were to be given when the urine output was more than $250 \mathrm{ml}$ per hour for consecutive 3 hours. The treatment resulted in a decrease in urine output from $165 \mathrm{mEq} / \mathrm{L}$ to $150 \mathrm{ml} /$ $\mathrm{hr}$, serum sodium of $160 \mathrm{mEq} / \mathrm{L}$. Her GCS improved from $7 / 15$ to $13 / 15$.

On the second day after admission, the patient developed a fever with her temperature ranging from $37.5^{\circ} \mathrm{C}$ to $39^{\circ} \mathrm{C}$. Progressive infiltrates were observed on chest radiographs taken over the next two days (Figure 1). A thick tracheal aspiration was noted and was sent for culture and sensitivity. Ventilator-associated pneumonia was confirmed. The culture of the tracheal aspiration revealed Klebsiella pneumoniae sensitive to polymyxin B and chloramphenicol and resistant to meropenem, amikacin, ampicillin, levofloxacin and imipenem.

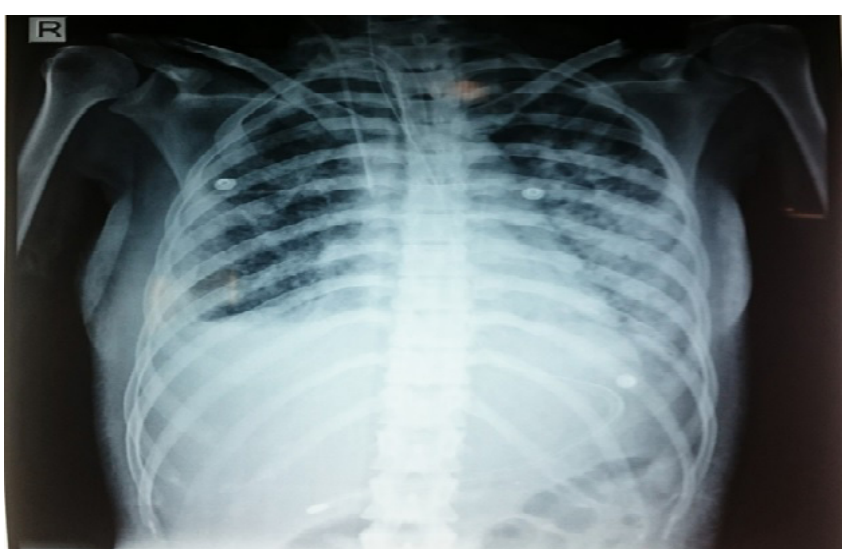

Fig. 1. Chest radiograph on the fourth hospital day. The image shows bilateral infiltrates due to ventilator-associated pneumonia. 
The patient subsequently showed a decrease in urine output, serum sodium and improvement in her GCS over the next period of five days.

Piperacillin-tazobactam was stopped, and on the fifth day after admission, the patient was prescribed polymyxin B (Health Biotech Limited, Chandigarh, India) $1,500,000$ units intravenously, then 1,000,000 units every twelve hours plus chloramphenicol (Biochem Pharmaceutical Industries, Mumbai, India, one gram every six hours.

With improving GCS and chest condition, she was extubated on the fifth day after admission and was moved from ICU on the sixth day after admission. Atropine was decreased by $10-20 \%$ every day and was stopped on the tenth days after admission. Her urine output (Figure 2) and serum sodium (Figure 3) were normal on the twelfth day after admission.

She was discharged from hospital on the fifteenth hospital day.

One week following discharge, she presented at the outpatient department for a follow-up. Her sodium and potassium levels were $138 \mathrm{mEq} / \mathrm{L}$ and $3.9 \mathrm{mEq} / \mathrm{L}$, respectively. She had also charted her urine output at her home, which was in the range of 2 to 2.5 litres/day.

\section{Discussion}

The neurologic manifestation of OP includes confusion, agitation, coma and seizure. The neurologic symptom of OP, atropine overdose and DI are similar that often leads to misdiagnosis. Diabetes insipidus in $\mathrm{OP}$ is rare, and the incidence is not known.

Diabetes insipidus can be central or nephrogenic. Our patient had central DI as the patient responded after desmopressin. There have been a few case reports of central DI following malathion [5], glufosinate [6], carbon monoxide [7] poisoning and overdose of baclofen and quetiapine [8] but there has been no case report following diethyl compound of OP. Central DI is common following pituitary surgery, tumour, infections, granulomas, inflammatory condition, vascular, chemical toxin and genetic but $50 \%$ is idiopathic [9], and our patient did not any of these causes.

Studies have shown that central DI usually appears within 24 hours of a CNS insult and lasts for 2-3 weeks. It rarely persists for more than three months [9]. Diagnosis is confirmed by urine and plasma osmolality, urine output and serum sodium levels.

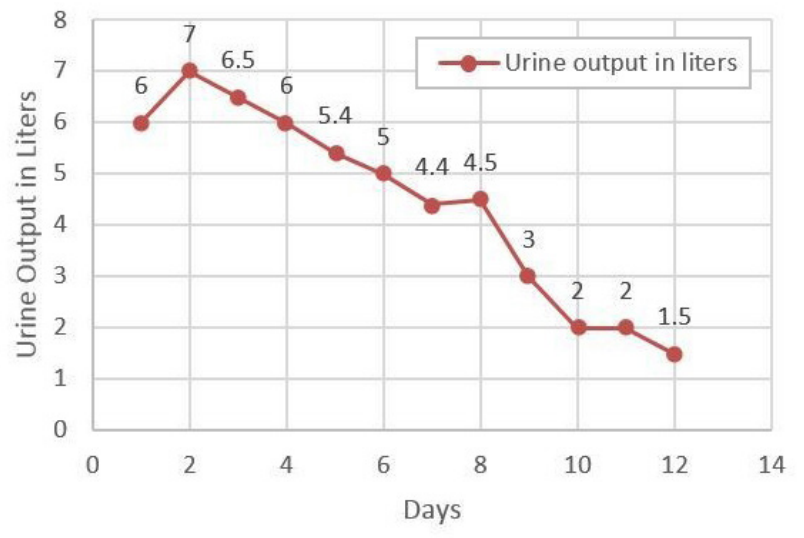

Fig. 2. Urine output during hospital stay

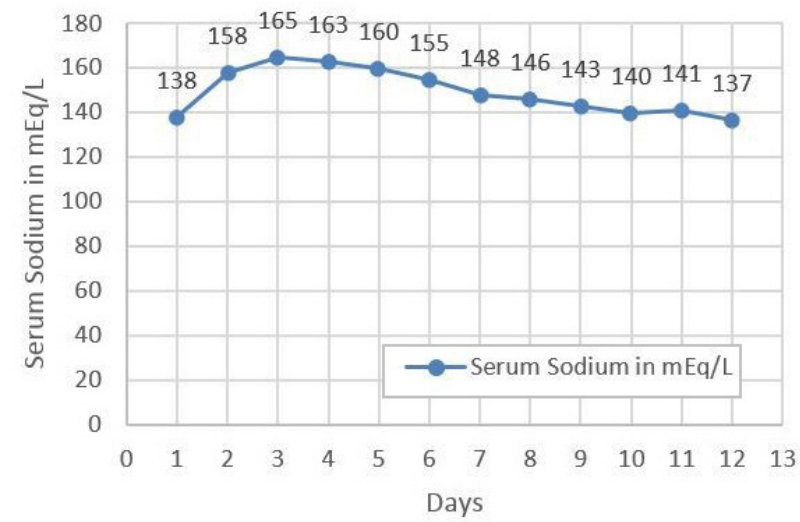

Fig. 3. Serum sodium during hospital stay

Mechanism of action of DI in OP poisoning is not known. The possible reason may be that OP may temporarily damage hypothalamus and pituitary, causing transiently decrease or absence of ADH. Therefore, a largescale study is required to explore the cause of DI in OP.

The treatment of central DI is to correct the underlying cause and fluid management. Studies have shown that vasopressin [10] can also be used instead of desmopressin, but vasopressin has a shorter duration of action than desmopressin, $5 \%$ dextrose or $0.5 \%$ normal saline is used for fluid management but in our patient 5\% dextrose was used.

\section{CONCLUSION}

Diabetes insipidus following organophosphorus poisoning is rare and requires a high degree of suspicion and timely referral to a higher centre in order to decrease mortality and improve patient outcome. 
148 - The Journal of Critical Care Medicine 2019;5(4)

\section{CONFLICT OF INTEREST}

None to declare.

\section{REFERENCES}

1. Bajracharya SR, Prasad PN, Ghimire R. Management of Organophosphorus Poisoning. J Nepal Health Res Counc. 2016;14(34):131-8.

2. Gyenwali D, Vaidya A, Tiwari S, Khatiwada P, Lansal DR, Giri S. Pesticide Poisoning in Chitwan, Nepal: a descriptive epidemiological study. BMC Public Health. 2017;17(1):619.

3. Thapa SR, Lama P, Karki N, Khadka SB. Pattern of poisoning cases in Emergency Department of Kathmandu Medical College Teaching Hospital. Kathmandu Univ Med J. 2008;6(2):209-13.

4. Nazima SN, Bashir Y, Nabi S, Bashir N.Intensive care management of organophosphorus poisoning patients: an experience from tertiary care center. International Journal of Advances in Medicine. 2018; 5(2):257-64.
Available online at: www.jccm.ro

5. Abdul-Ghaffar NU. Transient diabetes insipidus complicating severe suicidal malathion poisoning. J Toxicol ClinToxicol. 1997;35(2):221-3.

6. Takahashi H, Toya T, Matsumiya N, Koyama K. A case of transient diabetes insipidus associated with poisoning by a herbicide containing glufosinate. J Toxicol Clin Toxicol.2000;38(2):153-6.

7. UI Abideen Z, Mahmud SN, Rasheed A, Farooq Qasim Y, Ali F. Central Diabetes Insipidus and Hyperglycemia Hyperosmolar State Following Accidental Carbon Monoxide Poisoning. Cureus. 2017; 9(6):e1305.

8. Sileversides JA, Scott KC. Diabetes insipidus following overdose of baclofen and quetiapine. Anaesth Intensive Care 2009;37(2):319-20.

9. Saifan C, Nasar R, Mehta S, et al. Diabetes insipidus: a challenging diagnosis with new drug therapies. ISRN Nephro. 2013; 24:797620.

10. Qureshi S, Galiveeti S, Bichet DG, Roth J. Diabetes insipidus: celebrating a century of vasopressin therapy. Endocrinology. 2014;155(12):4605-21. 